\title{
Verhaltenstherapie bei depressiven Störungen im Alter
}

\author{
Gerald Gatterer
}

Online publiziert: 12. Dezember 2019

(C) Der/die Autor(en) 2019

\begin{abstract}
Zusammenfassung Depressive Störungen stellen neben organischen Störungen die Hauptgruppe der psychischen Erkrankungen im Alter dar. Ursachen hierfür sind einerseits die mit dem Alterungsprozess verbunden körperlichen, aber auch sozialen und kontextuellen Veränderungen und die Notwendigkeit der Verarbeitung derselben durch die betroffenen Menschen. Die vorliegende Arbeit stellt die Ursachen von depressiven Störungen im Alter und die Möglichkeiten der Verhaltenstherapie dar.
\end{abstract}

Schlüsselwörter Depression · Altern · Verhaltenstherapie

\section{Behavior therapy in old people sufferring from depression}

Summary Depression and organic disorders are the main groups of mental disorders in old age. Reasons for this are on the one hand the aging process and associated physical, but also social and contextual changes and the necessity of processing the same concerned by people. The present work represents the causes for depression in old people and the possibilities of behavior therapy in this area.

Keywords Depression · Aging · Behavior therapy

G. Gatterer $(\bowtie)$

Gerontopsychologisches Institut,

Schlossmühlgasse 22, 2351 Wiener Neudorf, Österreich

Institut für Alternsforschung, Sigmund Freud

Privatuniversität Wien, Freudplatz 1, 1020 Wien, Österreich

gerald@gatterer.at

\section{Einleitung}

Die Betreuung und Behandlung älterer Menschen mit Depressionen erfordert neben medizinischen und sozialen Maßnahmen auch psychotherapeutische Interventionen, um die mit dem Alter verbundenen Veränderungen und mögliche Krisen zu bewältigen (Rainer 2008). Dies beinhaltet die Auseinandersetzung mit Alternsveränderungen, Krankheiten, Verlusterlebnissen und verändertem Rollenverhalten. So ist etwa die Suicidrate bei älteren Menschen, vor allem älteren Männern, deutlich erhöht (Kapusta et al. 2007; Kapusta 2011). Obwohl zahlreiche Beiträge zur Gerontologie (Oswald et al. 2008) belegen, dass Altern nicht einseitig als Abbau von Funktionen, Fähigkeiten und Fertigkeiten angesehen werden kann, können andererseits die Tatsache der Multimorbidität und das Ansteigen psychischer Krankheiten (Förstl 2003) bei den über 60-jährigen nicht geleugnet werden.

Psychotherapie ist hier aber noch immer eine sehr vernachlässigte Fachdisziplin (Gatterer 1996, 2007, 2008; Maerker 2014). Offensichtlich sind viele PsychotherapeutInnen einer Lehrmeinung Freuds treu geblieben, der 1905 schrieb:

Das Alter der Kranken spielt bei der Auswahl der psychoanalytischen Behandlung insofern eine Rolle, als bei Personen nahe an oder über 50 Jahre einerseits die Plastizität der seelischen Vorgänge zu fehlen pflegt, auf welche die Therapie rechnet - alte Leute sind nicht mehr erziehbar - und als andererseits das Material, welches durchzuarbeiten ist, die Behandlungsdauer ins Unabsehbare verlängert. (Freud 1905, S. 21)

Diese Sichtweise erscheint heute nicht mehr angebracht. Seit den 80er Jahren des vorigen Jahrhunderts haben VertreterInnen der Psychoanalyse in Deutschland diese „leichtfertige Aussage Sigmund Freuds“ 
Abb. 1 Menschenbild der Verhaltenstherapie. (Mod. Gatterer 2013)



(Sophie Freud 2000, S. 310) ernsthaft hinterfragt und psychoanalytische Konzepte der Alternspsychotherapie entwickelt (siehe z.B. Radebold 1997; Radebold 1992; Bäurle et al. 2000). In den USA begann diese Entwicklung Anfang der 1960er Jahre. Das „Journal of Geriatric Psychiatry“ dient seit 1969 als psychoanalytisch orientierte geronto-psychiatrische Publikation. Trotzdem ist aber nach Hirsch (2000, S. 282) die Psychotherapie Älterer noch immer „durch viele Hindernisse eingeschränkt bzw. verunmöglicht“.

Die Ursachen der Vernachlässigung psychotherapeutischer Maßnahmen im Alter können nach Gatterer (2007) exemplarisch auf solche, die durch die TherapeutInnen selbst (Defizitmodell des Alterns, Angst vor dem eigenen Altern, vor Krankheiten, ... ), die Methode (Anpassung und Modifikation) oder den PatientInnen (somatisches Krankheitsmodell, Angst als verrückt zu gelten, ...) bedingt sind, zusammengefasst werden.

\section{Allgemeine Aspekte der Psychotherapie im Alter}

Altern ist ein multifaktorielles Geschehen, welches für den älteren Menschen vermehrt Anpassungsprozesse an sich verändernde Gegebenheiten (körperlich, sozial, Umwelt) zur Folge hat. Das Menschenbild der Verhaltenstherapie (Gatterer 2013; Abb. 1) geht davon aus, dass das Verhalten von Menschen das Resultat multifaktorieller Lernprozesse ist. Biologische Faktoren (Nerven, Gehirn, Neurotransmitter, etc.) stellen die biologische Grundlage dar. Lernprozesse führen in weiterer Folge zur Ausformung einer Persönlichkeit (Denkprozesse, emotionale Muster, Bindungsstile, Beziehungsmuster, Rollen, ...), die sich mit sozialen Faktoren auseinandersetzen muss (soziales Lernen von z.B. Werten und Normen der Gesellschaft). Weiters haben Umweltfaktoren und kontextuelle Faktoren (Licht, Kleidung, Hilfsmittel, Geld etc.) einen modulierenden Einfluss. Das endgültige motorische, kognitive, emotionale und soziale Verhalten ist dann das Resultat dieses Prozesses. Dabei entwickeln sich einerseits automatisierte Verhaltensweisen (multifaktorielle Skripts), die etwa $80 \%$ unseres Lebens beeinflussen. Weiters sind jedoch auch ständig neue Lernprozesse notwendig, um sich an neue Lebenssituationen anzupassen. Medizinische und psychologische Untersuchungen zum Altern haben gezeigt (Oswald et al. 2008), dass die Flexibilität und Plastizität des Gehirns abnimmt (verminderte Speed-Funktionen), jedoch die gut eintrainierten Verhaltensweisen sehr stabil bleiben. Dies gilt auch für biologische Prozesse wie schnelle biologische Verarbeitung von Reizen und Anpassung des Körpers an neue Situationen. Depressionen sind oft die Folge eines nicht geglückten Bewältigungsprozesses. Im Gegensatz zur Behandlung bei jüngeren Menschen, die oft mehr Ressourcen aufweisen, müssen bei der Auswahl der Probleme und Therapieziele bzw. der entsprechenden Methoden beim älteren Menschen deshalb folgende Faktoren vermehrt berücksichtigt werden: 
- Der biologische Aspekt: Krankheiten sowie körperliche Veränderungen nehmen im Alter vermehrt zu. Insofern kann deren Relevanz auch für psychologische und psychotherapeutische Maßnahmen nicht geleugnet werden. Hierzu zählen auch Veränderungen in der Tätigkeit der Neurotransmitter, die Belastbarkeit, aber auch die Funktionsfähigkeit der Sinnesorgane.

- Der biographische Aspekt: Nicht verarbeitete biographische Konfliktsituationen können im Alter wieder aktualisiert werden und dazu beitragen, dass noch vorhandene Entwicklungspotentiale nicht genützt werden und dadurch die Auseinandersetzung mit dem Alter erschwert wird. Dazu gehören etwa Traumatisierungen (Vergewaltigung, Ängste), Rollenbilder, Werte und Normen und fehlende Ausbildung.

- Der situative Aspekt: Im Alter entstehen oft auch neue Konflikte und Belastungen, die sich aus der individuellen Lebenssituation ergeben. Dazu gehören z. B. Krankheiten, der Verlust von Angehörigen sowie Änderungen in der Lebenssituation und den Rollen.

- Der personale Aspekt: Thomae (1996) konnte in seinen Untersuchungen feststellen, dass die subjektive Sicht der Vergangenheit, der Gegenwart und der Zukunft zentrale Dimensionen der individuell erlebten Welt darstellen und wesentlichen Einfluss auf den Alterungsprozess haben. Insofern spielen die eigenen Werte, Normen, Einstellungen etc. auch bei der psychotherapeutischen Behandlung eine wesentliche Rolle.

- Der soziale Aspekt: Soziale Kontakte stellen einen wichtigen Aspekt bei der Auseinandersetzung mit den Aufgaben und Anforderungen des Alterns dar. Oft ist hier eine Neuorientierung und ein Umlernen notwendig, um sich neuen Situationen adäquat anzupassen.

- Der kontextuelle Aspekt: Neben den bisher beschriebenen Faktoren kann der positive Alterungsprozess auch durch Umweltfaktoren negativ beeinflusst werden. So können Entwicklungspotentiale des älteren Menschen durch das Fehlen finanzieller Mittel, schlechte Wohnverhältnisse oder Krankheit behindert werden.

- Der systemische Aspekt: Oft sind einzelne Faktoren für sich allein nicht so relevant, jedoch deren $\mathrm{Zu}$ sammenspiel.

\section{Depression im höheren Lebensalter}

Psychische Störungen steigen im Alter ab 60 Jahren signifikant an, wobei Demenzerkrankungen und Depressionen am häufigsten auftreten. Der Anteil von depressiven Störungen liegt bei der Gruppe der über 70 -jährigen bei etwa $26 \%$ (Rainer 2008), wobei mittelschwere (13,8\%) und leichte Formen (12,4\%) überwiegen. Die Erfassung der Schwere der depressiven
Symptomatik erfolgt meist mittels der Geriatrischen Depressionsskala (Yesavage et al. 1983).

Bei affektiven Störungen im Alter ist in den meisten Fällen eine multifaktorielle Genese anzunehmen. Generell ist hierbei ein Zusammenspiel von biologischen, psychischen, sozialen und kontextuellen Faktoren gegeben (Gatterer 2013).

Bei affektiven Störungen lassen sich diese global folgendermaßen darstellen:

Organische Faktoren wie etwa Krankheiten, Veränderungen der Neurotransmitter (vor allem Serotonin), Nebenwirkungen von Medikamenten, Schmerzen, Gebrechlichkeit, aber auch Beeinträchtigungen beim Sehen und Hören stellen biologische Grundbedingungen dar (Niklewski und Baldwin 2003), auf denen psychologische Verarbeitungsprozesse aufbauen, die affektive Störungen zur Folge haben können. Diese sind abhängig von der Persönlichkeit des Betroffenen, dessen Werten, Normen und Einstellungen, Verstärkerprozessen, kognitiven und emotionalen Prozessen und auch übergeordneten Schemata des Denkens und Fühlens (vgl. Beck 2004; Young et al. 2005). Weiters spielen soziale Umgebungsbedingen bei der Krankheitsentstehung, der Verarbeitung und dem Verlauf eine wesentliche Rolle. Gerade bei Depressionen sind aber auch kontextuelle Variablen, wie etwa Lichtverhältnisse, die Stimulation durch die Umwelt und sonstige Lebensbedingungen, von Bedeutung.

Die wichtigsten, ursächlich für eine Depression in Betracht kommenden körperlichen Erkrankungen sind nach Haupt (2004) Hirnerkrankungen, entzündliche Erkrankungen, kardiopulmonale Erkrankungen, endokrine und metabolische Störungen, Neoplasien und Drogen bzw. Medikamente. An Demenz Erkrankte weisen, je nach Erhebungsmodus der Studie, in 30 bis $60 \%$ depressive Symptome auf (Haupt 2004).

Weiters können Schmerzen das Wohlbefinden und die Lebensqualität von Menschen erheblich beeinträchtigen. Im Rahmen der österreichischen Gesundheitsbefragung gaben je nach Alter 50 bis $62 \%$ der Frauen und 44 bis $47 \%$ der Männer erhebliche Schmerzen an. Häufigste Lokalisation war bei beiden Geschlechtern die Lendenwirbelsäule (jeweils mehr als $40 \%$ ), gefolgt von den unteren Extremitäten (Oberschenkel, Knie, Unterschenkel) (https://goeg.at/sites/ goeg.at/files/2017-06/seniorenbericht_2012.pdf).

Die psychosozialen Ursachen einer Depression im Alter können sehr vielfältig sein. In etwa $50 \%$ sind es belastende Lebensereignisse, die eine depressive Störung auslösen (Niklewski und Baldwin 2003). Hierzu zählen Verwitwung, fehlende soziale Kontakte sowie soziale Netzwerke und die oft damit verbundene Vereinsamung.

Als Risikofaktoren für die Entwicklung einer depressiven Symptomatik im Alter gelten (zitiert nach Niklewski und Baldwin 2003): 
Abb. 2 Genesemodell zur Enstehung von Verhaltensweisen und Störungen. (Mod. Gatterer 2013)

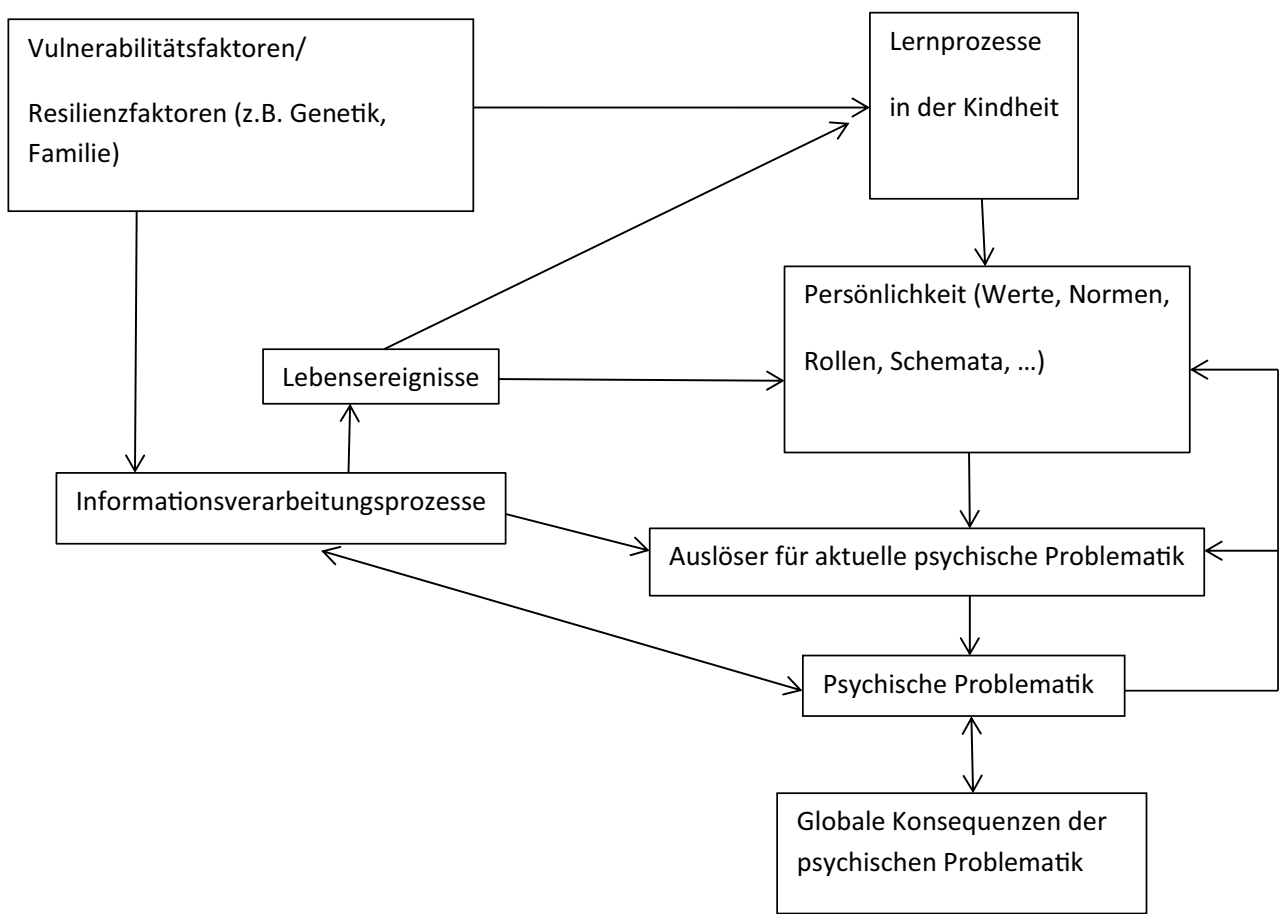

- weibliches Geschlecht,

- Persönlichkeitstyp (vor allem ängstlich vermeidend, zwanghaft, introvertiert-kontaktarm),

- frühere Depression,

- schwere körperliche Erkrankungen,

- Verwitwung und Scheidung.

Die Entwicklung einer depressiven Symptomatik kann mitunter durch den persönlichen Lebensstil beeinflusst werden. Ein aktiver Lebensstil mit regelmäßiger körperlicher Aktivität kann protektiv wirken, während SeniorInnen mit einem inaktiven Lebensstil eine deutlich erhöhte Prävalenz depressiver Störungen aufweisen (Niklewski 2006).

Zusätzlich spielen auch Umweltfaktoren, wie die Lichtverhältnisse, das Ausmaß von Stimulation, das Vorhandensein von Unterstützungsmaßnahmen, die finanzielle Situation und die Wohnverhältnisse, eine wesentliche Rolle (Bartova L. et al. 2019).

\section{Verhaltenstherapeutisches Vorgehen}

Im Vordergrund eines verhaltenstherapeutischen Behandlungsprogramms steht die genaue Erfassung der psychischen, kognitiven, somatischen, sozialen und ökologischen Ausgangslage zur Aufdeckung funktionaler Bedingungszusammenhänge zwischen diesen Faktoren. Dabei wird versucht, ein Erklärungsmodell für das Entstehen der Krankheit, ihr Weiterbestehen sowie Möglichkeiten zur Veränderung zu entwickeln. Grundsätzlich spielen bei der Genese der Depression genetische Faktoren, aber auch Vereinsamung und Verluste eine wesentliche Rolle. Das Problemverhalten (Symptomatik) wird dabei möglichst genau auf allen Ebenen (Verhalten, Kognitionen, physiologische Prozesse, Emotionen) beschrieben. Zum Verständnis des Entstehens von Verhaltensweisen (Genese der Störung) kann ein übergreifendes Modell verwendet werden (Abb. 2; mod. Gatterer 2013).

Prinzipiell ist der diagnostische Prozess darauf ausgerichtet, die aktuelle Symptomatik durch das Erfassen von Auslösern, prädisponierenden und Vulnerabilitätsfaktoren, der Persönlichkeit der/des Betroffenen, der Lebensgeschichte, aber auch den Konsequenzen (aufrechterhaltende Bedingungen) zu erklären und mit der/dem Betroffenen zu diskutieren. Aus verhaltenstherapeutischer Sicht können bei Depressionen im Alter das Modell der gelernten Hilflosigkeit (Seligman 1979), der Verstärkerverlust, sowie kognitive Verarbeitungsprozesse und Schemata, die das Verhalten und Empfinden massiv beeinflussen, zur Erklärung herangezogen werden. Als intervenierende Variablen müssen auch biologische und kontextuelle Faktoren berücksichtigt werden. Weiters wird das aktuelle Verhalten genau in seinem Ablauf erfasst. Zudem erfolgt eine kurze Abklärung der kognitiven Leistungsfähigkeit, um dementielle Erkrankungen frühzeitig zu erfassen und in den therapeutischen Prozess zu integrieren. Darauf bauen die geplanten Interventionen auf.

Die Vorteile des verhaltenstherapeutischen Vorgehens bei älteren Menschen mit Depressionen liegen in

- seiner Gegenwartsorientiertheit,

- der Anwendbarkeit durch paraprofessionelle und andere Berufsgruppen, 
Abb. 3 Therapeutische Maßnahmen bei Depressionen im Alter. (Mod. Gatterer 2013)

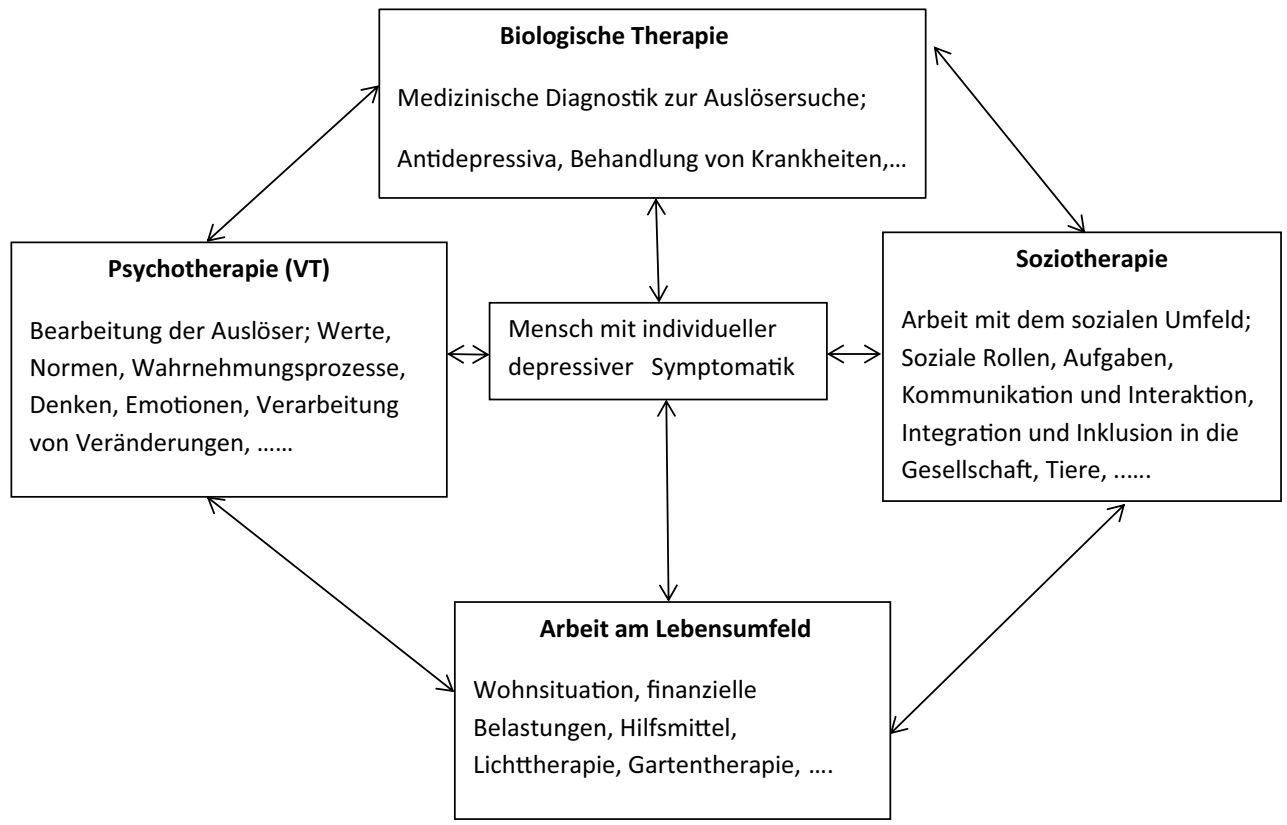

- der Integration von Techniken in den Klinikalltag bzw. Alltag der Betroffenen (z. B. Entspannungstechniken),

- der Möglichkeit, komplexe Verhaltensziele in einzelne Teilziele zu zerlegen und dadurch raschere Erfolgserlebnisse zu vermitteln,

- der Möglichkeit direkter und kontinuierlicher Beobachtung von Effekten,

- der damit gegebenen relativ guten Voraussetzungen zur Erfolgskontrolle,

- der Verfügbarkeit eines breiten Methodenrepertoires, welches verschiedensten Gruppen älterer Menschen gerecht werden kann. Die Anwendungsmöglichkeiten erstrecken sich hierbei von in physischer und psychischer Hinsicht kaum oder gar nicht beeinträchtigten Personen bis zu in allen Bereichen (kognitiv, psychisch, somatisch) schwerstgestörten geriatrischen Patienten,

- der Möglichkeit der Kombination mit anderen Therapieformen (Pharmakotherapie, pflegerischen Maßnahmen, Soziotherapie, Milieutherapie ....).

Verhaltenstherapeutische Interventionen bei Menschen mit Depression im höheren Lebensalter berücksichtigen sowohl biologische, psychologische, soziale und Umweltfaktoren (Abb. 3).

a) Genaue Verhaltensanalyse und Aufdecken von Ressourcen: Hier geht es vor allem um die genaue Ursachenanalyse, deren mögliche Beseitigung und das Aufgreifen von Ressourcen. Gerade ältere Menschen denken sehr defizitorientiert. Insofern sollte der Focus auf Ressourcen gelegt werden.

b) Aktivitätsaufbau bzw. Aktivitätstraining: Depressionen sind durch einen Mangel an Aktivität charakterisiert bzw. besteht ein direkter Zusammenhang zwischen dem Aktivitätsniveau und der Stimmung der/des Betroffenen. In der Therapie wird versucht, vorhandene Aktivitäten zu nützen bzw. neue langsam aufzubauen. Wesentlich ist dabei, die Patientin/den Patienten nicht zu überfordern.

c) Wahrnehmungstraining: Depressive sind dadurch charakterisiert, dass ihr Denken und ihre Wahrnehmung negativ gefärbt sind. Durch gezielte Übungen zur Wahrnehmung (sehen, hören, riechen, schmecken, tasten) soll eine realitätsbezogene Sicht ermöglicht werden. Dazu gehören auch achtsamkeitsbasierte Techniken z. B. Achtsamkeitsspaziergang

d) Kognitives Umstrukturieren: Verhaltenstherapie geht davon aus, dass negative Gefühle durch verzerrte Denkstrukturen bedingt sind. Dadurch kommt es zu einer negativen Sicht von sich selbst, der Umwelt, der Gegenwart und der Zukunft. In der Therapie werden diese kognitiven Grundannahmen, z. B. alles ist schlecht, überprüft und neue Denkprozesse gebahnt.

e) Werte, Normen, Einstellungen und Rollenbilder müssen im Rahmen des Alterungsprozesses oft neu überdacht und angepasst werden. Gelingt dies nicht, z. B. beim Auszug der Kinder, entsteht etwa das „empty nest syndrome“. Insofern ist es wesentlich, hier neue Lebensphilosophien zu entwickeln.

f) Arbeit an übergeordneten kognitiven Schemata: Schemata sind übergeordnete Denkstrukturen, die das Verhalten, Denken und Fühlen massiv beeinflussen. So kann etwa das Schema „Nur Menschen, die etwas leisten, sind etwas wert!" im Falle einer Krankheit und damit verbundener verminderter Leistungsfähigkeit zu Depressionen führen. In der Therapie werden diese Schemata aufgedeckt (z. B. durch den Schemafragebogen von Young), hin sichtlich ihrer Gültigkeit überprüft und neue Denk- 
muster erarbeitet, z. B. „Meine Familie liebt mich wie ich bin, auch wenn ich nichts leiste!". Für ältere Menschen wurde hier eine Kurzform entwickelt (Gatterer und Blokesch 2017).

g) Genusstraining: Menschen mit Depression haben verlernt $\mathrm{zu}$ genießen. Insofern wird in der Therapie genießen mit allen Sinnen wieder geübt (vgl. Koppenhöfer 2004).

h) Selbstsicherheitstraining: Gerade ältere Frauen sind nach dem Tod des Mannes mit der neuen Lebenssituation und dem Aufbau neuer sozialer Kontakte überfordert. Viele Dinge hat der „Mann“ gemacht. Diese müssen deshalb oft neu erlernt werden. Vor allem der Aspekt „für sich selbst etwas tun“ ist eine neue Herausforderung.

i) Hierzu gehören auch Konzepte wie „individuelle“ neue Sinnfindung und neuen Aufgaben im Alter trotz aller Einschränkungen und Veränderungen. Etwas was vor allem Viktor Frankl (2005) betont hat.

j) Ergänzend können auch soziale Interventionen, körperliche Übungen und Sport, Entspannungstraining (Jacobson-Progressive Muskelentspannung) bzw. Paartherapie durchgeführte werden.

k) Klinisch psychologische Behandlungsprogramme (z.B. kognitives Training) sind vor allem bei Menschen, die Angst haben „dement“ zu werden, sinnvoll.

l) Veränderungen in den Lebensbedingungen z.B. Wohnsituation, technische Hilfsmittel, Gartentherapie, Tiere als Therapie und bedürfnisorientierte Modelle ergänzen die verhaltenstherapeutischen Interventionen.

m) Eine medikamentöse Therapie mittels moderner Antidepressiva kann unterstützend eingesetzt werden bzw. erscheint bei schweren Depressionen indiziert.

Über die genaue Anwendung verhaltenstherapeutischer Verfahren, wie etwa operante Methoden, Aufund Abbau von Verhalten, Verstärkung, kognitive Umstrukturierung und andere psychische Erkrankungen wird auf die entsprechende Literatur verwiesen (Gatterer 2007, 2016; Margraf und Schneider 2018; Hautzinger 2016).

\section{Praktisches Beispiel}

Frau M., 76 Jahre, kommt wegen einer leichten depressiven Symptomatik in die psychotherapeutische Praxis. Diese besteht seit dem Tod des Mannes vor 2 Jahren. Damals sei es ihr sehr schlecht gegangen und sie nehme auch Antidepressiva und ein Schlafmittel. Da es ihr aber noch immer nicht gut gehe, möchte sie auch eine Psychotherapie machen. Die Anamnese ergibt, dass Frau M. sehr glücklich verheiratet war. Sie hat einen Sohn und einen Enkel. Diese sehe sie alle 2 Wochen. Sie selbst schildert sich als sehr ordentlich, bindungsorientiert, familienorientiert und brave Hausfrau, Mutter, Großmutter und früher auch Ehe- frau. Die Familie sei für sie sehr wichtig. Sie habe nur eine gute Freundin, da sie ja die Familie habe. Weggehen würde sie eigentlich nicht, außer Sonntag in die Kirche. Dort treffe sie auch Bekannte. Aktuell leide sie noch immer unter dem Tod des Mannes und Einsamkeit. Er gehe ihr sehr ab. Es freue sie deshalb auch nicht zu kochen, da es sich für eine Person nicht auszahle. Wenn die Kinder kommen gehe es ihr viel besser. Sie habe auch Schlafstörungen, da ihr ihr Mann besonders am Abend fehle, wenn sie allein im Bett liegt. Im Schema-Kurzfragebogen hatte sie hohe Werte in den Bereichen „Verstrickung/soziale Abhängigkeit; Verlassenheit; subjektive Inkompetenz; Selbstaufopferung und Selbstbestrafung“. Aus verhaltenstherapeutischer Sicht kann ihre Depression (vereinfacht) so erklärt werden, dass Frau M. bereits in ihrer Kindheit eine starke Familienorientierung und Abhängigkeit gelernt hat. Sie ist sehr rollenkonform erzogen worden und ist deshalb sehr stark in ihrer Rolle Mutter, Ehefrau und Hausfrau verwurzelt, die ihr nun fehlen (Rollen- und Funktionsverlust; Verstärkerverlust). Wenig entwickelt ist ihre Rolle selbständige Frau, mit den eigenen Bedürfnissen, die gelernt hat sich selbst zu verwöhnen. Insofern erschien es notwendig, ihre Rolle als Witwe neu zu definieren. Dazu gehörte die Akzeptanz des Todes des Ehemannes, die Verarbeitung der Trauer, die innere Erlaubnis zur Definition einer Witwenrolle, die auch wieder glücklich sein darf. Ebenso der Aufbau einer neuen Frauenrolle, die über die Rolle Hausfrau, Mutter, Großmutter und Ehefrau hinausgeht. Ebenso war der Aufbau neuer Verhaltensweisen, wie etwa Genusstraining, soziale Aktivitäten, alte und neue Freunde treffen, Urlaub mit der Freundin, Integration in der sozialen Kommune etc. wichtig. Wahrnehmung und Kommunikation wurden vermehrt auf positive Faktoren gerichtet. Dadurch war es der Patientin wieder möglich, Spaß und Lebensfreude zu entwickeln, ohne das Gefühl zu haben, dadurch ihren Mann zu vergessen, den sie mehrmals die Woche am Friedhof besuchte und Gespräche führte, was sie alles erlebt hatte. Das galt auch für die Kommunikation mit dem Sohn, der Schwiegertochter und dem Enkel, mit denen sie vermehrt Kontakte über das Smartphone und neue Medien (E-Mail, WhatsApp) hatte, dadurch auch Fotos, Videos und Erlebnisse austauschen konnte und somit wieder mehr Anteil an deren Leben und am Leben ganz allgemein nahm. Die Therapie gestaltete sich anfangs schwierig, da stark emotional besetzte Themen reflektiert und auch in Frage gestellt wurden. Nach 15 Sitzungen, verbunden mit einem vom Therapeuten begleiteten Friedhofsbesuch und einem „therapeutischen“ Gespräch mit dem Ehemann und wie er sich ihr Leben und ihre neue Rolle vorstellen würde, konnte sie erstmals eine neue Lebensphilosophie entwickeln. Sie bekam von ihm ,indirekt“ die Erlaubnis, wieder am Leben teilzuhaben, ohne eine böse Frau zu sein, die den Ehemann nicht geliebt hatte. Dadurch waren auch die weiteren Schritte der Therapie mit Genuss, Spaß und Freude möglich. Insgesamt 
dauerte die Therapie 38 Sitzungen, bis zur $24 \mathrm{~h}$ wöchentlich, dann 14-tägig. Die letzten 5 Sitzungen erfolgten im Abstand von 3 Wochen bzw. die letzte nach einem Monat zur Stabilisierung.

\section{Zusammenfassung und Ausblick}

Die vorliegende Arbeit versucht den Einsatz verhaltenstherapeutischer Methoden bei älteren Menschen mit depressiver Symptomatik praktisch darzustellen. Im Vordergrund aller dieser Interventionen steht:

a) eine Stärkung der Kompetenz des älteren Menschen zur Bewältigung seines Alltags mit größtmöglicher Selbständigkeit und Unabhängigkeit und

b) der Erhalt bzw. eine Erhöhung der (subjektiven) Lebensqualität durch Veränderung der somatischen, ökonomischen, ökologischen und sozialen Bedingungen.

Gerade aus dieser Zieldefinition ist jedoch auch ersichtlich, dass Psychotherapie im höheren Lebensalter nicht isoliert von anderen Therapien (medikamentöse Therapie, Ergotherapie, Physiotherapie, Logopädie, pflegerischen Maßnahmen, ...) gesehen werden kann, sondern einen Teil eines ganzheitlichen Therapieansatzes darstellt. Andererseits erscheint aber auch die Ausgliederung psychotherapeutischer Maßnahmen bei „rein medizinischen“ Therapien nicht Ziel führend.

Trotz dieser Möglichkeiten psychotherapeutischer Maßnahmen im höheren Lebensalter erfordert es jedoch weitere Bemühungen und vor allem ein Umdenken in der Gesellschaft, bei den Betroffenen und bei den TherapeutInnen und ÄrztInnen. Letztere müssen sich vermehrt mit den Bedürfnissen älterer Menschen beschäftigen und ihren Wissensstand über die Forschungsergebnisse der modernen Gerontologie, insbesondere der Gerontopsychologie und Sozialgerontologie, erweitern.

Es ist eine Philosophie geworden, den älteren Menschen möglichst lange am Leben zu erhalten. Insofern erscheint es aber notwendig, ihn auch seelisch-geistig am Leben zu erhalten.

Interessenkonflikt G. Gatterer gibt an, dass kein Interessenkonflikt besteht.

Open Access Dieser Artikel wird unter der Creative Commons Namensnennung 4.0 International Lizenz (http:// creativecommons.org/licenses/by/4.0/deed.de) veröffentlicht, welche die Nutzung, Vervielfältigung, Bearbeitung, Verbreitung und Wiedergabe in jeglichem Medium und Format erlaubt, sofern Sie den/die ursprünglichen Autor(en) und die Quelle ordnungsgemäß nennen, einen Link zur Creative Commons Lizenz beifügen und angeben, ob Änderungen vorgenommen wurden.

\section{Literatur}

Bartova, L., et al. (2019) Depression. Medikamentöse Therapie. Konsensus Statement. State of the Art2019. ClinicumNeu- ropsy. Sonderausgabe November 2019. https:// oegpb.at/ wp-content/uploads/2019/11/Konsensus_Depression_ 2019_08.11.pdf.Zugegriffen:02.12.2019.

Bäurle, P., et al. (2000). Klinische Psychotherapie mit älteren Menschen. Bern:Huber.

Beck, A.T. (2004). Kognitive Therapie der Depression. Herausgegeben von Martin Hautzinger. Aus dem Amerikanischen von Gisela Bronder (3. Aufl.). Beltz-Taschenbuch: Psychologie. Weinheimu. a.: Beltz.

BMG (2012). Gesundheit und Krankheit der älteren Generation. https://goeg.at/sites/goeg.at/files/2017-06/ seniorenbericht_2012.pdf.Zugegriffen:02.12.2019.

Förstl,H. (Hrsg.). (2003). Lehrbuch der Gerontopsychiatrie und - psychotherapie. Grundlagen - Klinik-Therapie(2.Aufl.). Stuttgart, NewYork: Thieme.

Frankl, V.E. (2005). Der Wille zum Sinn (5. Aufl.). Bern: Huber.

Freud, S. (1905). über Psychotherapie, GW V(S.11-26).

Freud, S. (2000). Lohnt sich Psychotherapie mit alten Menschen? In: Klinische Psychotherapie mit älteren Menschen, Bäurle, etal. (Hrsg.), (S. 307-310). Bern: Huber.

Gatterer, G. (1996). Psychosomatische Störungen im höheren Lebensalter. In P. Fischhof \& H.G. Zapotoczky (Hrsg.), Handbuch für Gerontopsychiatrie. Wien: Springer.

Gatterer, G. (Hrsg.). (2007). Multiprofessionelle Altenbetreuung. Bd. 2. Wien-New York: Springer.

Gatterer, G. (2008). Depressionen im Alter. Facharzt, 5, 18-22.

Gatterer, G. (2013). Integrative Verhaltenstherapie im Alter. PsyMed, 3(24), 45-58.

Gatterer, G. (2016). Gerontopsychiatrische Erkrankungen. In Elsevier - Altenpflegekonkret. Gesundheits- und Krankheitslehre5. Aufl. München: Urban \& Fischer.

Gatterer, G., \& Blokesch, R. (2017). Need oriented schemata and schema-therapy in old aged people. Presentation European Congress of Psychology. Amsterdam. http:// orbilu.uni.lu/bitstream/10993/31769/1/Symposium_ GeroPsychology_2017.pdf.Zugegriffen: 02.12.2019.

Haupt, M. (2004). Depressive Störungen im Alter - Symptombesonderheiten und körperliche Erkrankungen. Zeitschrift für Gerontopsychologie \& -psychiatrie, 17(4), 215-224.

Hautzinger, M. (2016). Depression im Alter: Psychotherapeutische Behandlung für das Einzel- und Gruppensetting. Mit E-Book inside und Arbeitsmaterial. Weinheim: Beltz.

Hirsch, R. D. (2000). Anforderungen an eine Weiterbildung für Psychotherapie mit älteren Menschen. In P. Bäurle, et al. (Hrsg.), Klinische Psychotherapie mit älteren Menschen. Bern:Huber.

Kapusta, N. D., Etzersdorfer, E., \& Sonneck, G. (2007). Trends in suicide rates among the elderly in Austria 1970-2004: an analysis of changes by age group, suicide method and gender. Int J Geriatric Psychiatry, 22(5), 438-444.

Kapusta, N.D. (2011). Aktuelle Daten zur Zahl der Suizide in Österreich. http://www.suizidforschung.at/statistik suizide_oesterreich.pdf.Zugegriffen:02.12.2019.

Koppenhöfer, E. (2004). Kleine Schule des Genießens. Lengerich: Pabst.

Maerker, A. (Hrsg.). (2014). Alterspsychotherapie und klinische Gerontopsychologie. Heidelberg: Springer.

Margraf, J., \& Schneider, S. (2018). Grundlagen, Diagnostik, Verfahren und Rahmenbedingungen psychologischer Therapie. Bd. 1.Berlin: Springer.

Niklewski, K. (2006). Depression. In W.D. Oswald, U. Lehr, C. Sieber \& J. Kornhuber (Hrsg.), Gerontologie (Bd. 3, S. 48-55). Stuttgart: Kohlhammer.

Niklewski, G., \& Baldwin, B. (2003). Depressive Erkrankungen. In H. Förstl (Hrsg.), Lehrbuch der Gerontopsychiatrie und 


\section{übersichtsarbeit}

-psychotherapie. Grundlagen - Klinik - Therapie (2. Aufl. S. 436-448). Stuttgart, New York: Thieme.

Oswald, W.D., Gatterer, G., \& Fleischmann, U. M. (2008). Gerontopsychologie (2. Aufl.). Wien: Springer.

Radebold,H. (1992). Psychodynamik und Psychotherapie Älterer. Berlin:Springer.

Radebold, H. (Hrsg.). (1997). Altern und Psychoanalyse. Göttingen:Vandenhoeck\&Ruprecht.

Rainer, M. (2008). Depression im Alter. Psychopraxis, 3, 12-16.

Seligman, M.E.P. (1979). Erlernte Hilflosigkeit. München, Wien, Baltimore: Urban und Schwarzenberg.

Thomae, H. (1996). Das Individuum und seine Welt. Eine Persönlichkeitstheorie. Göttingen: Hogrefe.

Yesavage, J. A., Brink, T. L., Rose, T. L., etal. (1983). Development and validation of a geriatric depression screening scale: a preliminary report. J. Psychiatr. Res., 39, 37-49.

Young, J. E., Klosko, J. S., \& Weishaar, M.E. (2005). Schematherapie. Ein praxisorientiertes Handbuch. Paderborn: Junfermann.

Hinweis des Verlags Der Verlag bleibt in Hinblick auf geografische Zuordnungen und Gebietsbezeichnungen in veröffentlichten Karten und Institutsadressen neutral. 